This is a author version of

F. Haizmann, D. Schlipf, S. Raach, A. Scholbrock, A. Wright, C. Slinger, J. Medley, M. Harris, E. Bossanyi and P. W. Cheng,

"Optimization of a Feed-Forward Controller Using a CW-lidar System on the CART3,"

published in Proceedings of the American Control Conference, Chicago, USA, 2015.

\title{
Optimization of a Feed-Forward Controller Using a CW-lidar System on the CART3
}

\author{
Florian Haizmann ${ }^{1}$, David Schlipf ${ }^{1}$, Steffen Raach ${ }^{1}$, Andrew Scholbrock ${ }^{2}$, Alan Wright ${ }^{2}$, \\ Chris Slinger ${ }^{3}$, John Medley ${ }^{3}$, Michael Harris ${ }^{3}$, Ervin Bossanyi ${ }^{4}$, Po Wen Cheng ${ }^{1}$
}

\begin{abstract}
This work presents results from a new field-testing campaign conducted on the three-bladed Controls Advanced Research Turbine (CART3) at the National Renewable Energy Laboratory in 2014. Tests were conducted using a commercially available, nacelle-mounted continuous-wave lidar system from ZephIR Lidar for the implementation of a lidar-based collective pitch feed-forward controller. During the campaign, the data processing of the lidar system was optimized for higher availability. Furthermore, the optimal scan distance was investigated for the CART3 by means of a spectra-based analytical model and found to match the lidar's capabilities well. Throughout the campaign the predicted correlation between the lidar measurements and the turbine's reaction was confirmed from the measured data. Additionally, the baseline feedback controller's gains were tuned based on a simulation study that included the lidar system to achieve further load reductions. This led to some promising first results, which are presented at the end of this paper.
\end{abstract}

\section{INTRODUCTION}

During the last few years, several contributions from the scientific community have proposed lidar-assisted control concepts to reduce fatigue loads of modern wind turbines, ranging from collective pitch feed-forward [1], [2], individual pitch control [3], nonlinear model predictive control [4], [5], [6], and yaw control [7], [8]. In spite of this, only very few results from field testing of such concepts have been reported so far. As a follow-up to the campaigns conducted by Stuttgart Wind Energy (SWE) and the National Renewable Energy Laboratory (NREL) in 2012 [9], [10], a new field testing campaign was arranged by NREL, DNV GL, ZephIR, and SWE. The campaign used a commercial lidar from ZephIR that was mounted on the three-bladed Controls Advanced Research Turbine (CART3) to have the ability to further test and optimize different controllers.

For this work, NREL provided the CART3 as well as the operation and data acquisition system on-site, ZephIR provided a continuous-wave $(\mathrm{CW})$ lidar system, and DNV GL and SWE each provided their controllers. Despite this, in this work only the SWE controller is considered. ZephIR adjusted the lidar to optimize the system's data availability

Part of this research was funded by the German Federal Ministry for the Environment, Nature Conservation and Nuclear Safety (BMU) in the framework of the German joint research project "LIDAR II".

${ }^{1}$ Stuttgart Chair of Wind Energy (SWE), Universität Stuttgart, Germany, Florian.Haizmann/David.Schlipf/Steffen.Raach

(at) ifb.uni-stuttgart.de

${ }^{2}$ National Renewable Energy Laboratory (NREL), USA, Andrew. Scholbrock/Alan.Wright (at) nrel.gov

${ }^{3}$ ZephIR Lidar, UK, Chris.Slinger/John.Medley/ Michael.Harris (at) zephirlidar.com

${ }^{4}$ DNV GL, UK, Ervin.Bossanyi (at) dnvgl.com and meet the requirements for using the data for real-time control. SWE investigated the optimal scan distance for the lidar combined with its feed-forward controller by using a spectra-based analytical model [11] of the correlation between the rotor-effective wind speed sensed by the turbine and its lidar estimate. In addition, the SWE controller was optimized by re-tuning the feedback gains in a simulation study. This study included the simulation of a lidar that has characteristics that are very similar to the real system's characteristics.

With these optimizations the controller was run for a couple of minutes, and the findings are provided below. These results confirm the expected correlation between the lidar and the turbine and show the potential for reducing loads.

This paper is organized as follows. Section II summarizes the field-testing environment. Section III outlines the different optimizations. Section IV presents the campaign results, and Section $\mathrm{V}$ discusses the conclusions and future work.

\section{FiELD-TESTING ENVIRONMENT}

\section{A. The CART3}

The field testing for this work was conducted on NREL's CART3 at the National Wind Technology Center in Boulder, Colorado. The geographical location and the easy access to the turbine's control system make the CART3 an optimal test environment for evaluating new and advanced control algorithms of wind turbines. The CART3 is a $600-\mathrm{kW}$ variable-speed pitch-controlled turbine with a rotor diameter of $40 \mathrm{~m}$, a hub height of $36.6 \mathrm{~m}$, and a rated rotor speed of $37 \mathrm{rpm}$. It was modified with a three-bladed rotor and as a result, currently operates at $550 \mathrm{~kW}$ because of resonance issues [12]. Heavy instrumentation, such as strain gauges, accelerometers, and a meteorological tower-installed $80 \mathrm{~m}$ in front of the turbine's mean wind direction $\left(292^{\circ}\right)$ complemented the field-testing setup.

\section{B. The ZephIR DM Lidar System}

The ZephIR DM is a circularly scanned, CW coherent Doppler lidar ${ }^{1}$. Optimized for installation on a nacelle roof, it generates line-of-sight (LOS) Doppler measurements every $20 \mathrm{~ms}$. The period of the continuous circular scan is $1 \mathrm{~s}$. Research reported by Bossanyi [1] indicates that this lidar scan geometry and frequency is very well suited to lidar feed-forward turbine control. The LOS measurements are

\footnotetext{
${ }^{1}$ WWW. zephirlidar.com/DM
} 


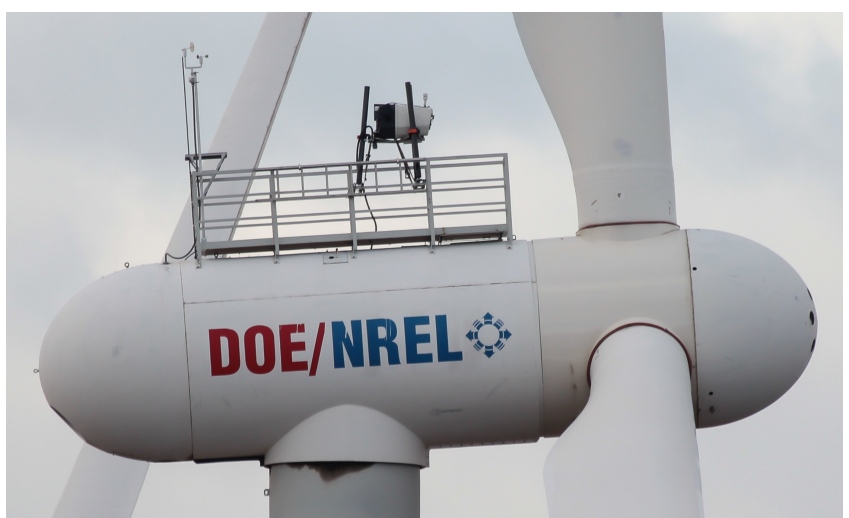

Fig. 1. The ZephIR DM lidar installed on the CART3 at the National Wind Technology Center. (Photo by Lee Jay Fingersh, NREL 32910)

used by the ZephIR to calculate various wind field quantities, such as horizontal wind speed, vertical wind shear, wind veer, and wind yaw misalignment relative to the turbine axis. The lidar can operate at ranges from $10 \mathrm{~m}$ to more than $300 \mathrm{~m}$. CW operation and the focusing approach to probe beam localisation ensures high sensitivity, permitting measurements even in very clear air, as encountered during the field-testing campaign reported here.

Figure 1 shows the ZephIR lidar installed on the CART3.

\section{The SWE Collective Pitch Feed-Forward Controller}

The tested controller used in this study is the same as in the previous campaigns in 2012 and is described in more detail in [9] and [10]. Because of the modular concept of the SWE collective pitch feed-forward controller, only the module "LidarDataProcessing" had to be changed to incorporate the signals from the ZephIR DM lidar. The rotor-effective wind speed from lidar data is calculated by:

$$
v_{0 L}=v_{\text {horz }} \cos \alpha_{H},
$$

where $v_{\text {horz }}$ and $\alpha_{H}$ are the horizontal wind speed and the yaw misalignment from the ZephIR output, respectively. The calculation is done this way because the longitudinal wind component is assumed to be responsible for the aerodynamic torque. Furthermore, the quality flags reported by the system with every measurement are evaluated to ignore bad data.

\section{OptIMIZATION}

To achieve the best results possible with this field-testing campaign, different optimizations regarding the lidar system's algorithms, its measurement configuration, and the feedback controller have been performed.

\section{A. Lidar System Algorithms}

The lidar determines the incoming wind field by combining multiple LOS wind speed measurements in various ways. To generate the required rotor-effective measurements, the lidar's "fit-derived" algorithms were used. These algorithms apply a parameterised wind model in real time, using a least-squares fitting procedure, to the measured LOS Doppler readings. As a result, horizontal wind speed at the centre of the scan, wind yaw misalignment, and the power law coefficient of vertical shear can be obtained. During the experiments, these wind measurements were updated at 100-ms intervals, which were accessed using the MODbus protocol for input into the controller. The ZephIR DM lidar was initially optimized to provide highly accurate 10min-averaged wind measurements, as might be required for conventional turbine performance measurements. However, control systems require maximum signal availability at high rates. Therefore, during the experimental period reported here, the lidar's data filters were adjusted to maximise availability, ensuring reliable inputs to the controller at up to $0.1 \mathrm{~Hz}$. Availability (as defined by "lidar quality" in Section IV) was greater than $98.5 \%$ after these optimizations.

\section{B. Lidar System Measurement Configuration}

To get good lidar measurements, an optimization of the physical measurement configuration is needed.

For the given lidar system, only the measurement distance can be optimized, because the system comes with a fixed circular trajectory at a fixed cone half-angle of $15^{\circ}$ and an averaging time of $2 \mathrm{~s}$ (corresponds to two sweeps around the circle trajectory) for the "fit-derived" outputs. Those values have been fixed before deploying the lidar system.

Basically, there are two issues to be considered when optimizing a lidar's measurement configuration for feedforward control. First, the measurement distance should be chosen to get the best correlation. This is a challenging task, because there are several effects involved that change the correlation, such as wind evolution and the range weighting function. At a closer distance, the wind will evolve less, but the range weighting will be smaller. Furthermore, the rotor extracts the most energy at around $3 / 4$ of the blade span, and the wind speed at this position will contribute more to the rotor-effective wind speed. Second, the signal has to be transferred in a timely fashion to the control system. To avoid unnecessary and harmful control action and obtain optimal performance, the wind speed from the lidar has to be filtered by an adaptive low-pass filter based on the transfer function $G_{R L}$ between the rotor-effective wind speed and its lidar estimate [13], [14]. Thus, a minimum distance can be calculated to have a timely control action.

Here, we need for each measurement distance $x_{L}$ the transfer function $G_{R L}$ to calculate the time delay introduced by the filtering. This can be done by conducting a simulation study in the time domain, as shown in Figure 2. Therefore, the wind components $u, v$, and $w$ on a spatial and temporal grid $(x, y, t)$ have to be generated by inverse Fourier transformations that are based on spectral properties. In the case of the Kaimal turbulence model, these spectral properties are the auto spectrum $S_{i i, h}$ for the wind component $h$ of the $i$ th grid point and the squared coherence $\gamma_{i j, 1}^{2}(k)$ between the longitudinal component of the $i$ th and the $j$ th grid point [15]. Then, the wind field has to be scanned by a lidar simulator to obtain the LOS wind speeds, and from those the lidar estimate of the rotor-effective wind speed $v_{0 L}$ is 


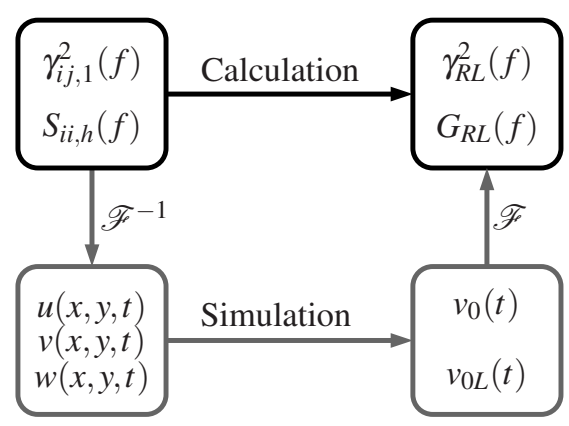

Fig. 2. Basic concept of the correlation model.

calculated. Furthermore, the rotor-effective wind speed $v_{0}$, which is based on simulated turbine data or directly from the wind field, has to be calculated. In the last step, the transfer function $G_{R L}$ and the squared coherence $\gamma_{R L}^{2}$ can be estimated based on Fourier transformations. Because of the properties of the numerical Fourier transformation the estimate of the transfer function improves with the length of the simulation. Finally, we would need to conduct endless simulations to get exact values.

To avoid this issue, we solved this constrained problem with a brute force optimization based on a spectra-based analytical model [11], shown Figure 2. The main idea is to calculate the correlation directly based on the turbulence spectral properties. The following three steps give a short overview of the applied procedure:

1) Correlation Coefficient: One way to quantify the quality of correlation between the rotor-effective wind speed and its lidar estimate is to use the correlation coefficient:

$$
\rho_{R L}=\frac{\sigma_{R L}^{2}}{\sigma_{L} \sigma_{R}}
$$

where $\sigma_{R L}^{2}$ is the covariance between the two signals $v_{0}$ and $v_{0 L}$, and $\sigma_{L}$ and $\sigma_{R}$ are the standard deviations, respectively. For perfect correlation, $\rho_{R L}$ is 1 and for no correlation, 0 .

The value of $\rho_{R L}$ can be obtained in the time domain from the two signals without any problems. In the frequency domain, we can use the following relationships:

$$
\begin{aligned}
\sigma_{R}^{2}=\int_{-\infty}^{+\infty} S_{R R} \mathrm{~d} f, \sigma_{L}^{2} & =\int_{-\infty}^{+\infty} S_{L L} \mathrm{~d} f \\
\text { and } \sigma_{R L}^{2} & =\int_{-\infty}^{+\infty} S_{R L} \mathrm{~d} f
\end{aligned}
$$

where $S_{R L}$ is the cross spectrum between both signals, and $S_{L L}$ and $S_{R R}$ are the auto spectra. The spectra can be calculated by the Fourier transforms of the signals as described in [11]. Wind evolution is considered in the analytical model by using the model from [16] and the decay parameter $\alpha=0.4$. Ongoing research shows that this parameter is site dependent and might be too high to represent the real wind evolution.

Finally, we can calculate the correlation coefficient $\rho_{R L}$ directly from the spectral wind information for each measurement distance $x_{L}$, as shown in Figure 3 (top). Hence, if only considering $\rho_{R L}$, the optimal measurement distance would be $x_{L}=40 \mathrm{~m}$.
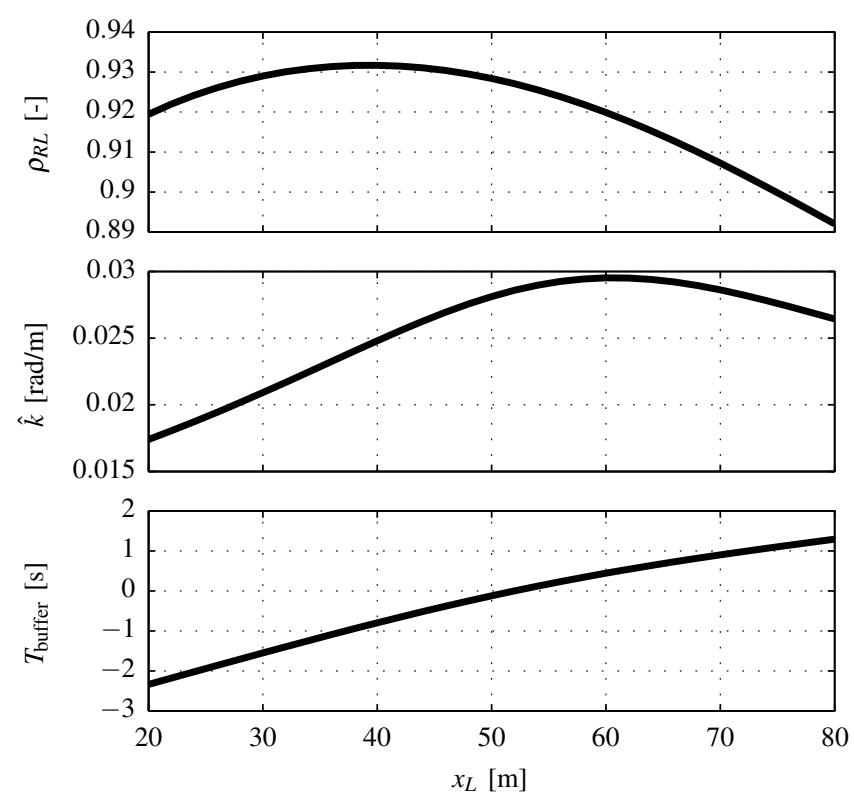

Fig. 3. Correlation coefficient (top), maximum coherent wave number (center), and buffer time (bottom) over measurement distance.

2) Timing: As mentioned earlier, the timing issue also has to be addressed to find the optimal measurement distance. Therefore, the time delay of the low-pass filter fitted to each transfer function $G_{R L}$ has to be calculated. The transfer function $G_{R L}$ from the lidar estimate of the wind speed to the rotor-effective wind speed can be calculated by:

$$
G_{R L}=\frac{S_{R L}}{S_{L L}} .
$$

Because of its low-pass behaviour, the transfer function is approximated by a first-order Butterworth filter. The filter is parametrized by a cut-off frequency, $f_{\text {cutoff }}=\hat{k} \bar{v} /(2 \pi)$, at $-3 \mathrm{~dB}$ below the static gain, where $\hat{k}$ is the maximum coherent wave number and $\bar{v}$ is the average wind speed.

The time delay is obtained from the following considerations: with Taylor's hypothesis, the wind needs the time $x_{H} / \bar{v}$ to evolve from the measurement plane to the hub of the turbine. Because of the averaging over the full trajectory, $v_{0 L}$ is already delayed by $T_{\text {Scan }} / 2=1 \mathrm{~s}$. The filter delay is approximated by $T_{\text {Filter }}$. When using the filtered wind in the feed-forward controller, the signal has to be synchronized with the wind reaching the rotor plane, as shown in Figure 4. Therefore, the necessary time delay is:

$$
T_{\text {Buffer }}=\frac{x_{H}}{\bar{v}}-\frac{1}{2} T_{\text {Scan }}-T_{\text {Filter }}-\tau .
$$

The time $\tau$ compensates the pitch actuator, which is negligible because the CART3's pitch actuators are very fast.

Finally, the maximum coherent wave number $\hat{k}$ and the corresponding $T_{\text {Buffer }}$ can be calculated for each focus distance $x_{L}$, shown in Figure 3 (center and bottom). We used $\bar{v}=20 \mathrm{~m} / \mathrm{s}$, assuming that we would not operate the CART3 at higher mean wind speeds. Compared to the two lidar systems used in 2012, the maximum coherent wave number 


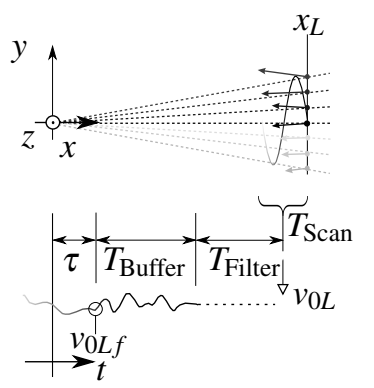

Fig. 4. Timing of the lidar signal.

$\hat{k}$ is lower for the ZephIR DM lidar than for the SWE scanner [10], but similar to the one for the Vindicator [9].

3) Results: A negative buffer time $T_{\text {Buffer indicates that }}$ the wind speed signal would be too late. We can define the constrained optimization problem as maximizing the correlation coefficient $\rho_{R L}$ by changing $x_{L}$ such that the buffer time $T_{\text {Buffer }}$ is not negative:

$$
\max _{x_{L}} \rho_{R L} \quad \text { such that: } T_{\text {Buffer }} \geq 0 .
$$

Therefore, the optimal focus distance considering both optimal correlation and timely signal is $x_{L}=52 \mathrm{~m}$. Because this is close to the suggested value by ZephIR for the measurement distance of $x_{L}=58 \mathrm{~m}$, no changes were made to this value.

\section{Re-Tuning of the Feedback Gains}

Embedding a feed-forward controller in the control loop offers the ability to re-tune the feedback gains. The bandwidth of the feedback controller can be lowered because the feed-forward controller is rejecting the main ratio of the disturbance. The feedback loop stabilizes the steady state and accommodates for improper disturbance measurements and model uncertainties. Thus, the obtained benefits of the feedforward controller can be reinvested in tuning the feedback gains to reduce structural loads.

A lidar feed-forward controller mainly assists the feedback controller in low frequencies because the lidar signal is filtered to avoid harmful control action. The improvement of the feed-forward controller can be observed in Figure 5 in the power spectral density (PSD) analysis of the generator speed. In a brute-force optimization, the feedback gains are tuned by relaxing the gains to obtain a high reduction in the damage equivalent loads (DELs). The gains are chosen such that a similar control performance as without the lidar feed-forward controller is achieved for the generator speed. The optimization was made using two different wind fields, with mean wind speeds of $16 \mathrm{~m} / \mathrm{s}$ and $18 \mathrm{~m} / \mathrm{s}$, and yielding a reduction of the proportional gain $k_{p}$ and an increased time constant $T_{i}$ of the integral controller.

To validate the obtained optimized feedback gains, a lifetime analysis (LTA) was performed and evaluated with respect to the DELs of the tower-base bending moment $M_{T y}$ and the standard deviation of the generator speed $\Omega_{g}$.
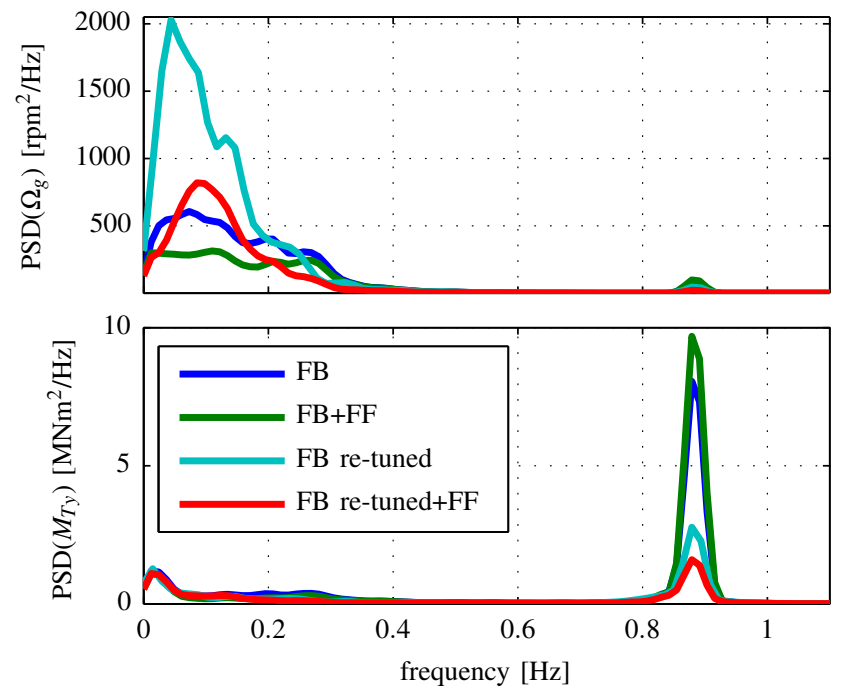

Fig. 5. PSD analysis of the generator speed $\Omega_{g}$ and the tower-base bending moment $M_{T y}$ of the compared controllers for a stochastic class B wind field with a mean wind speed of $16 \mathrm{~m} / \mathrm{s}$.

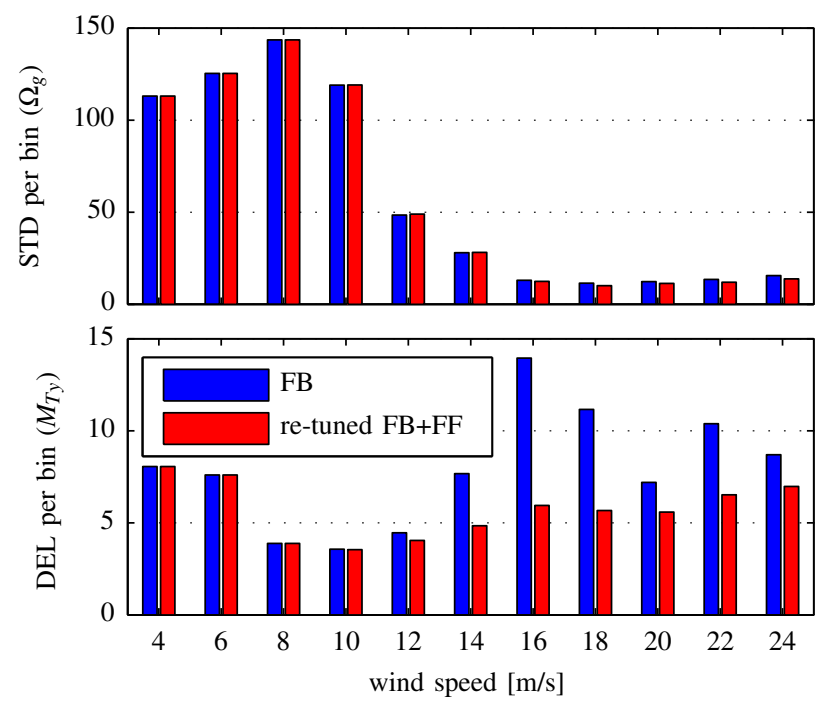

Fig. 6. Lifetime analysis for the baseline controller and the retuned baseline incorporated with the feed-forward controller.

Figure 6 shows the LTA comparison between the feedback controller and the retuned feedback controller incorporated with the lidar feed-forward controller. The standard deviation of the generator speed was similar for both controllers but the DEL was heavily reduced for the optimized controller setup.

\section{RESUlts}

\section{A. Verification of the Correlation}

During the campaign, the correlation was verified to design the SWE feed-forward controller. For this verification, we extracted two blocks of the CART3 data collected in the beginning of December 2013 (see Table I). The blocks were chosen according to the following criteria: 

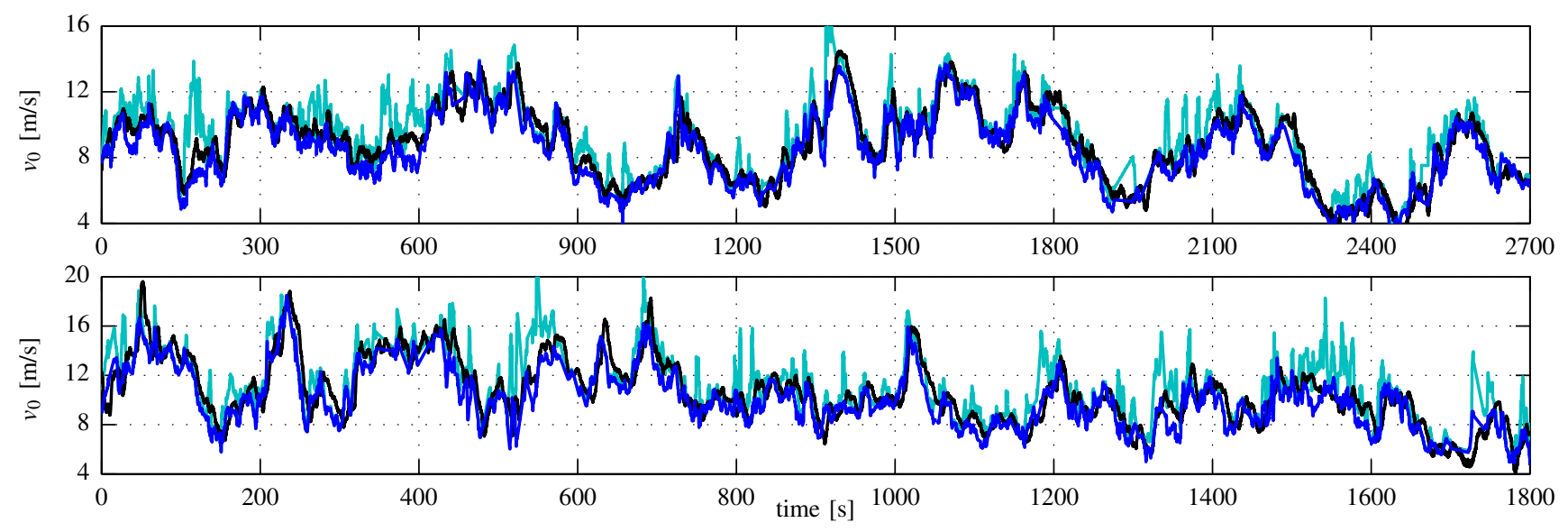

Fig. 7. Rotor-effective wind speed from turbine (black) and lidar data (dark blue) and horizontal wind speed (light blue). Block 1 (top) and 2 (bottom).

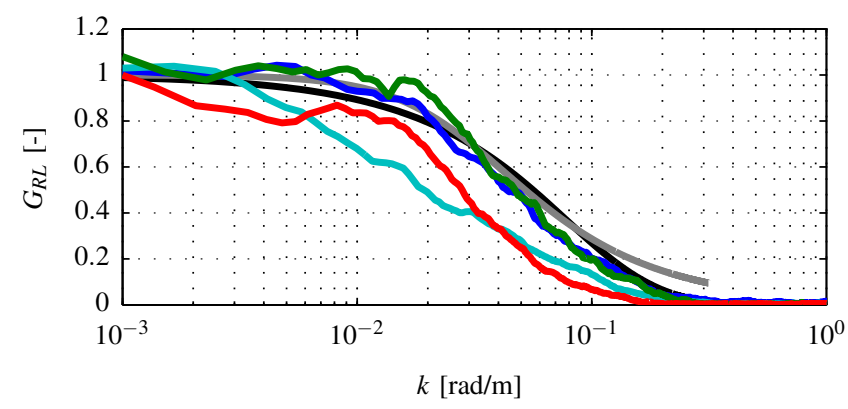

Fig. 8. Transfer functions: model (black). Data: between $v_{0}$ and $v_{0 L}$ (Block 1: dark blue; Block 2: green) and between $v_{0}$ and $v_{\text {horz }}$ (Block 1: light blue; Block 2: red). Fitted filter (gray).

- Wind speeds constantly over $4 \mathrm{~m} / \mathrm{s}$ are needed to apply the wind speed estimator using turbine data

- Block length must be at least $30 \mathrm{~min}$ to have enough data to calculate the spectra

- Lidar quality must be at least $60 \%$.

Lidar quality is defined as the percentage of the data that is flagged by the lidar system as a valid measurement (fit flag equal to 1 and no error code in the data itself). During the beginning of the campaign, this value was around $40 \%$, and sometimes no data were available for various consecutive seconds of the measurement. As discussed earlier, reoptimization of the ZephIR lidar's settings to maximise availability significantly improved the lidar data quality to above 90\% during later phases of the measurement campaign.

For both blocks, the horizontal wind speed, rotor-effective wind speed from the lidar, and turbine data are plotted in Figure 7. In both blocks, the calculated rotor-effective wind speed $v_{0 L}$ matches the reaction of the turbine $v_{0}$ better than the horizontal wind speed $v_{\text {horz }}$. This can be confirmed with the transfer functions calculated from the spectra of the data. In Figure 8, the measured transfer function from $v_{0 L}$ to $v_{0}$ fits the model better than the transfer function from $v_{\text {horz }}$ to $v_{0}$. This means that using $v_{0 L}$ instead of $v_{\text {horz }}$ for the feed-forward control requires less filtering and provides more benefits regarding the expected load reductions. Therefore, we used $v_{0 L}$ and set $\hat{k}=0.03 \mathrm{rad} / \mathrm{m}$.

Regarding the timing, we discovered that the measured wind arrives as expected at the turbine. We assumed that the lidar is installed around $2 \mathrm{~m}$ behind the hub and thus $x_{H}=x_{L}-2 \mathrm{~m}=56 \mathrm{~m}$ in front of the turbine. Based on this assumption, the expected delay between $v_{0 L}$ and $v_{0}$ should be with $T_{\text {Scan }}=2 \mathrm{~s}$ :

$$
T_{\text {Preview }}=\frac{x_{H}}{\bar{v}}-\frac{1}{2} T_{\text {Scan }}
$$

Because we measured values close to this (see Table I), no tuning was needed, and we kept the prediction time at $0 \mathrm{~s}$.

Based on this analysis, the correlation of the ZephIR lidar and the CART3 - predicted by the analytical model-could be confirmed. Hence, the timing and filtering of the SWE feed-forward controller is set to a prediction time of $\tau=0 \mathrm{~s}$ and a maximum coherent wave number of $\hat{k}=0.03 \mathrm{rad} / \mathrm{m}$.

\section{B. Field-Testing Results from the Optimized Controller}

Because of low wind conditions during the campaign, the CART3 was de-rated to a rated rotor speed of $26 \mathrm{rpm}$.

Unfortunately, still only a few minutes of region 3 data could be collected before the lidar system was dismounted; however, this data shows promising results, which indicate that the controller works as intended. Figures 9 and 10 show a reduction of the generator speed variations in the expected frequency range.

\section{Conclusions And Outlook}

This work presents results from the field testing of a commercial CW-lidar system as input to the SWE collective pitch feed-forward controller. Different optimizations have been successfully applied to the lidar system's internal algorithms and the measurement configuration of the system as well as to the gains of the feedback controller. The results show that using a commercial lidar system for feed-forward control is possible, and, the structural turbine loads can be reduced as intended. 
TABLE I

OVERVIEW OF EVALUATED DATA.

\begin{tabular}{c|c|c|r|r|r|r|r|r} 
Block & Start Time & End Time & \multicolumn{2}{|c|}{ Lidar } & Mean wind speed [m/s] & \multicolumn{2}{|c|}{ Delay [s] } & \multicolumn{2}{c}{$\hat{k}$} \\
ID & & & quality [\%] & $v_{0}$ & $v_{0 L}$ & Taylor & Data & {$[\mathrm{rad} / \mathrm{m}]$} \\
\hline 1 & $12-0101: 05: 00$ & $12-0101: 50: 00$ & 65.83 & 8.83 & 8.45 & 6.6 & 6.2 & 0.025 \\
2 & $12-0203: 55: 00$ & $12-0204: 25: 00$ & 67.94 & 10.64 & 10.12 & 5.5 & 5.4 & 0.031
\end{tabular}

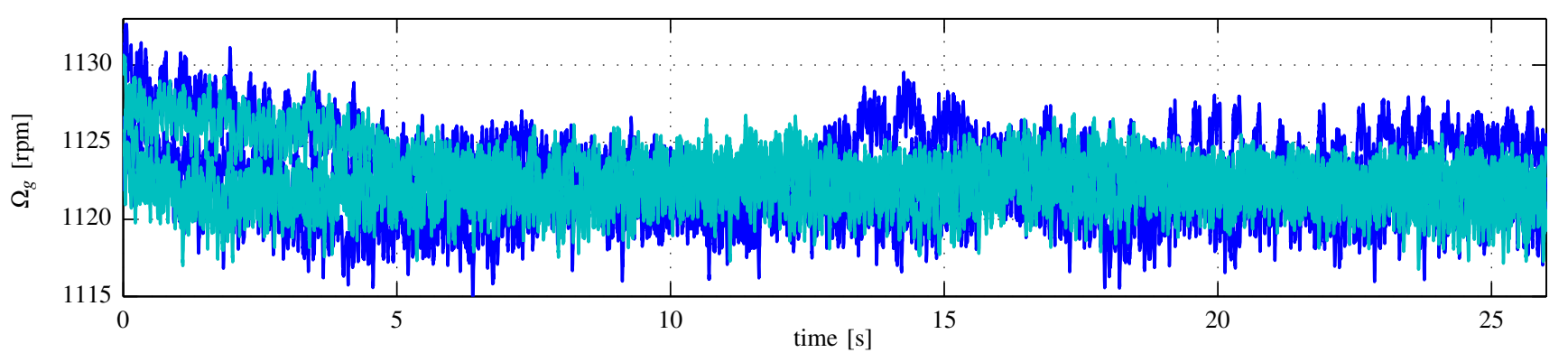

Fig. 9. Generator speed $\Omega_{g}$ from de-rated and re-tuned controller: FF off (dark blue) and FF on (light blue).

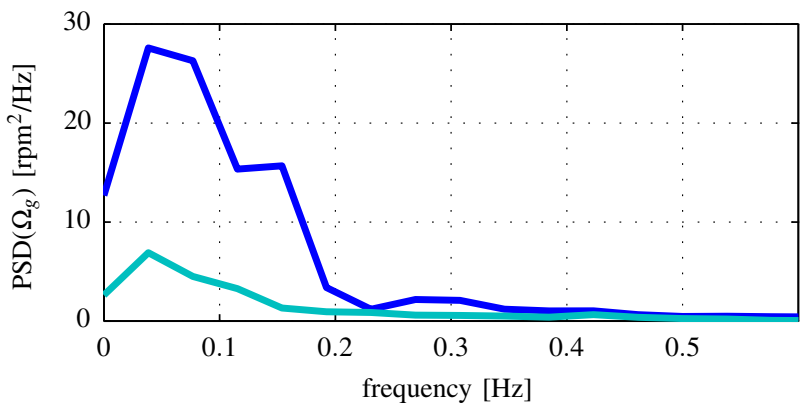

Fig. 10. PSD of generator speed $\Omega_{g}$ from the de-rated and re-tuned controller: FF on (dark blue) and FF off (light blue).

During the testing portion of the campaign, it was not possible to collect more than a few minutes of data. This means that more data has to be collected in future field-testing campaigns to provide more substantiated conclusions.

\section{ACKNOWLEDGMENTS}

The authors would like to thank everyone from NREL, SWE, ZephIR, and DNV GL involved in this campaign for their contribution and support. This work was supported by the U.S. Department of Energy under Contract No. DE-AC36-08GO28308 with the National Renewable Energy Laboratory. Funding for the work was provided by the DOE Office of Energy Efficiency and Renewable Energy, Wind and Water Power Technologies Office.

\section{REFERENCES}

[1] E. Bossanyi, A. Kumar, and O. Hugues-Salas, "Wind turbine control applications of turbine-mounted lidar," in Proceedings of The Science of Making Torque from Wind, Oldenburg, Germany, 2012.

[2] D. Schlipf and M. Kühn, "Prospects of a collective pitch control by means of predictive disturbance compensation assisted by wind speed measurements," in Proceedings of the German Wind Energy Conference DEWEK, Bremen, Germany, 2008.

[3] F. Dunne, D. Schlipf, L. Y. Pao, A. D. Wright, B. Jonkman, N. Kelley, and E. Simley, "Comparison of two independent lidar-based pitch control designs," in Proc. 50th AIAA Aerospace Sciences Meeting Including the New Horizons Forum and Aerospace Exposition, 2012.
[4] J. Laks, L. Y. Pao, E. Simley, A. Wright, N. Kelley, and B. Jonkman, "Model predictive control using preview measurements from LIDAR," in Proceedings of the 49th AIAA Aerospace Sciences Meeting Including the New Horizons Forum and Aerospace Exposition, Orlando, USA, 2011.

[5] A. Körber and R. King, "Nonlinear model predictive control for wind turbines," in Proceedings of the European Wind Energy Association Annual event, Brussels, Belgium, 2011.

[6] S. Gros, "An economic nmpc formulation for wind turbine control," in Decision and Control (CDC), 2013 IEEE 52nd Annual Conference on, Dec 2013, pp. 1001-1006.

[7] K. A. Kragh, M. H. Hansen, and T. Mikkelsen, "Precision and shortcomings of yaw error estimation using spinner-based light detection and ranging," Wind Energy, vol. 16, no. 3, p. 353Ü366, 2012.

[8] P. A. Fleming, A. K. Scholbrock, A. Jehu, S. Davoust, E. Osler, A. D. Wright, and A. Clifton, "Field-test results using a nacelle-mounted lidar for improving wind turbine power capture by reducing yaw misalignment," Journal of Physics: Conference Series, vol. 524, no. 1, p. 012002, 2014.

[9] A. Scholbrock, P. Fleming, L. Fingersh, A. Wright, D. Schlipf, F. Haizmann, and F. Belen, "Field testing LIDAR based feed-forward controls on the NREL controls advanced research turbine," in 51th AIAA Aerospace Sciences Meeting Including the New Horizons Forum and Aerospace Exposition, Dallas, USA, 2013.

[10] D. Schlipf, P. Fleming, F. Haizmann, A. Scholbrock, M. Hofsäß, A. Wright, and P. W. Cheng, "Field testing of feedforward collective pitch control on the CART2 using a nacelle-based lidar scanner," in Proceedings of The Science of Making Torque from Wind, Oldenburg, Germany, 2012.

[11] D. Schlipf, J. Mann, and P. W. Cheng, "Model of the correlation between lidar systems and wind turbines for lidar assisted control," Journal of Atmospheric and Oceanic Technology, vol. 30, no. 10, pp. 2233-2240, 2013.

[12] P. A. Fleming, A. D. Wright, L. J. Fingersh, and J.-W. van Wingerden, "Resonant vibrations resulting from the re-engineering of a constantspeed 2-bladed turbine to a variable-speed 3-bladed turbine," in Proceedings of the 49th AIAA Aerospace Sciences Meeting Including the New Horizons Forum and Aerospace Exposition, Orlando, USA, 2011.

[13] D. Schlipf and P. W. Cheng, "Adaptive feed forward control for wind turbines," at - Automatisierungstechnik, vol. 61, no. 5, pp. 329-338, 2013

[14] E. Simley and L. Pao, "Reducing lidar wind speed measurement error with optimal filtering," in Proceedings of the American Control Conference, Washington, USA, 2013.

[15] IEC 61400-1, Wind turbines - Part 1: Design requirements, International Electrotechnical Commission Std., Rev. third edition, 2005.

[16] R. A. Pielke and H. A. Panofsky, "Turbulence characteristics along several towers," Boundary-Layer Meteorology, vol. 1, pp. 115-130, 1970. 